\title{
A New Look at the Program Development Process
}

p. Hieman, IBM Boeblingen

This paper is a contribution to the discussion of

defining a program development process frame with distinct validation points for analysing and measuring process results

providing methods, techniques and tools to support the program development process.

\section{O. INTRODUCTION}

In the 25 years history of professional system programming, system programmers have developed systems of increasing complexity in function and size. Their experience shows that it is increasingly more difficult to meet specified system integrity/quality objectives without complying to disciplined programming methods based upon a firm definition of the program development process. System programmers have identified and developed a series of programming support techniques which are mandatory for producing and validating a high quality of specifications and code.

In the first section of this paper, a process frame with its validation points and some basic process measurements are described. The second section describes the methods supporting the program development process. The final section of the paper provides some proof regarding the quality of succeeding os releases that have been improved by applying new process support techniques. 
1. THE PROGRAMING PROCESS AND SOME MEASURES

Most people think of programming cycles as being composed of four activities (Figure 1):

Designing: Where it is determined what is needed and defined what the programing wi11 do and how it wi11 work.

Coding: Where the program is written.

Testing: Where it is verified that the program will work and problems uncovered during testing are fixed.

Integrating: Where the pieces or modules are put together to create an application, a subsystem, or an entire system.

While testing and integration are closely related in that integration has to take place before larger units of code can be tested, it is resource consuming if one has to go back to design during coding or even testing.

The results of coding, testing and integration are operational programs of growing size and complexity (Figure 2)

- Program Unit relates to the smallest self-contained piece of code. It may be a module, a macro, or a subroutine.

- A function is composed of program units. A function may be a command, a parameter, or a recovery routine.

- A component is composed of functions. A component may be an access method, a compiler, or the supervisor.

- The components are integrated to form a system, as VS/2 Release 1, OS Release 21, and so on. 
About two or three years ago, IBM started to analyse financial and statistical information relating to quality of systems. The customer was experiencing an APAR rate that was too high. Figure 3 shows the average number of all APARs for OS submitted per customer, in other words, al1 submitted APARs, divided by the number of customers. The area labeled Valid is the average number of valid APARs submitted per customer. A valid APAR is one that requires a fix either in code or in documentation. The difference between valid APARs and the total, largely represents duplicates and user errors. The average number of APARs submitted per user has not changed significantly over the past four releases, nor has the average number of valid APARs.

Corresponding to the high error rates the analysis showed that $76 \%$ of the cost for Release 18 was spent on maintenance and only 24 : was spent on development (Figure 4). Development includes cost related to designing and coding programs, to writing and printing publications, and to performing all testing and integration. Maintenance includes FE cost to find problems and laboratory costs to fix the problems.

The analysis also indicated that the cost of finding a problem increased as the programming process progressed. The cost of finding and fixing a problem after the system was released (an APAR) was thirty times the cost of fixing it in unit test, the first set of tests that the code goes through in development (Figure 5). The cost of finding a problem during coding is virtually nothing. As we progress trom unit test through field usage, the cost increases at each testing stage. More and more people are involved with the code as it moves through the cycle, and more people and their work are affected when a problem occurs.

The net result of the analysis was to establish an improved process that will help in:

- reducing errors

- finding problems as early as possible. 
The base of the new process was a frame of seven distinct phases with distinct validation points at the end of each phase (Figure 6). Validation regards to

- Quality of the results produced during one phase

- Progress in terms of goals and schedules

- Usefulness and Profitability of the system/component/function to be developed further.

The process should be continued to its next phase only, if predetermined validation criteria are met.

Phase 0 is a planning phase to develop requirements. These are statements about the functions needed to provide new or improved data processing capability. People study, analyze, and perhaps survey users to determine what they require.

Phase I determines the requirements for a particular programming package. It addresses configuration, storage requirements, performance, etc.

Phase II is the external design phase. User interfaces (for example commands, parameters, output formats) and the interfaces with major system components are developed.

Phase III is the internal design phase. The internal logic of the program, the module structure as well as internal data areas and interfaces are developed. During this phase, a test plan that covers all functional variations is also developed.

Phase 0 to III are strictly sequential steps; phase IV and $V$ the implementation and testing phases, however, interact with each other.

Nevertheless, Phase IV is the main coding phase including unit and function testing. 
Each phase has its own unique output or results:

- Documentation in the first 4 phases

- Code in Phase IV

- A system in Phase $V$

- Fixes in the maintenance phase VI

Besides the planning documentation, the following documents relate directly to the program being developed:

Market requirements define a need, independent of any programming considerations. Objectives state the requirements of a particular programming package. External specifications define the purpose of the program, its user interfaces, and its interfaces with other parts of the system. Internal specifications describe the $\operatorname{logic}$ and the method of operation of the program and its internal interfaces. Code is also considered as documentation since the field engineer receives source code documented in the form of microfiche.

2. THE METHODS SUPPORTING THE PROGRAMMING PROCESS

According to the phase structure of programing projects, there are

- methods supporting the programm development process

- methods and criteria to check the completeness of the results of a phase

In describing the methods, this paper distinguishes between

- Principles

- Tools and Techniques

- Management Aspects 


\section{$2.1 \quad$ Principles}

2.1.1 Top-Down Design

Top-down design is used today for most new development projects. In top-down design, we take a functional viewpoint. We define the basic functions of the program and design them with a high level of detail. Then each of those basic functions is broken into more detailed subfunctions. We move down through levels of detail, creating a tree structure like the one shown in Figure 7 . We continue this process until all subfunctions are defined to a consistent level of detail. When we are finished with the topdown design process, we will know about all of our interfaces, all of our logic decisions, and how the data is structured. Each box in the tree structure represents a function and, in most cases, a code segment, an inline block of code, or a subroutine that does not exceed a listing page.

Top-down design avoids simultaneous, inconsistent interface definition. Top-down design has reduced the complexity of the design and of the programs that result from the design. It also provides for orderly logic development. When design is done from the bottom up, decisions may be assumed to be in the upper-level logic, when in fact they should happen in the bottom levels.

\subsubsection{Structured Programs (Figure 8)}

To reflect the program structure that has been developed during design, we emphasize on development of structured code. Structured code is composed of one-page segments. Control always enters a segment at its top and leaves at the bottom. Al1 segments are referenced by name. Go Tos are not permitted. Each segment eithor returns to its caller or moves inline to the next segment. 
Some advantages of structured code are:

- It is easy to read. A listing of a program in structured code can be read and understood very quickly. This is often impossible if the program is written using traditional coding methods.

- Structured code is easier to maintain and to extend. A programmer can learn structured coding in about a week of coursework and practice; and once he has learned it, he generally wil1 not go back to traditional methods.

\subsubsection{Elements of Structured Code (Figure 9)}

Structured coding uses basic program elements to code any program. By using simple building blocks for sequential statements, IFTHEN-ELSE, and DO-WHILE, we can simplify programming, reducing complexity.

\subsubsection{Continuous Integration (Figure 10)}

As mentioned earlier, code development implies the integration of code pieces of growing size and complexity into the system. We use a process called continuous Integration. Functions are integrated into the system as they become available according to a disciplined plan. "Disciplined" means that functions must be added in a logical sequence, and that resources must be devoted to the right functions at the right time. We start integration early, for large efforts as much as eighteen months before shipment. A driver is a subsystem used for subsequent testing. Drivers are built by continually adding functions. If the entire system followed top-down design and implementation, then one can take the integration plan directly from the top-down plan. 
Here are some of the advantages of continuous integration:

- Code is entered into the system when it is complete. Small sets of modules are added at one time, instead of trying to put all the modules together at once, $i$.e. the integration process is less complex.

- A real system is always running, and it is easier to keep it running.

- Detailed planning becomes necessary. Programmers are forced to think through their dependencies so that their interfaces will become more apparent. It also forces people to become more realistic in their scheduling.

2.1.5 Tests \& Test Criteria (Figure 11)

We distinguish between four types of tests according to the integration levels:

- Unit Test

- Function Test

- Component Test

- System Test

Unit testing is done by the developer after his code is completed. It tests the smallest self-contained piece of code, for example a module, a macro, or a subroutine. The minimum criterion for completion is that ail the branches in the code are executed both ways.

Function test occurs after unit test is completed. In function test, we put units together to form discrete functions, for example a command, a recovery procedure, or a parameter. The criterion or completion is $100 \%$ execution of a11 test cases, $90 \%$ of hich must be successful. 
Component test takes place after function test is completed. Functions are put together to form a component. A component may be an access method, the supervisor, or a compiler. The criterion for completion is $100:$ of all test cases were executed successfu11y.

The final test is system test. The components are put together to form the system, for example OS 20.1 or VS/2 Release 1.

Criteria for completion are all test cases executed successfully and all problems fixed. Whatever criteria is used by other development groups, it is important that predetermined criteria are used throughout the whole process (including specification validation) and should never be compromised.

\section{$2.2 \quad$ Tools and Techniques}

This section describes techniques and tools that support programmers to perform their tasks according to established principles.

\subsubsection{HIPO (Figure 12)}

We are supporting the design task by a technique called HIPO that in turn is supported by a tool that provides of updating and print facilities.

HIPO stands for Hierarchy, plus Input, Process, Output. Hierarchy means proceeding from very generalized to very detailed drawing, following top-down design practices. The diagrams refer to successively lower-level diagrams.

Each diagram shows the inputs to a function, the processing steps within the function, and the outputs. The data and function involved in the process, therefore, becomes visible. HIPOs is a replacement for flowcharts. 


\subsubsection{Cause / Effect Graph}

Due to the absence of formal design languages, we describe program functions in prose. Unfortunately prose text has the characteristic of burying relationships, i.e. to make relationships less visible. A technique of analyzing external specifications is to draw a cause and effect graph. A cause and effect graph is a Boolean representation of the logical relationships documented in specifications. Figure 13 shows an example of a graph representing some specifications of how to construct a valid expression. These graphs help to find errors early, such as omissions and logical inconsistencies. They provide a measure and a structure for a thorough review of a specification.

\subsubsection{Test Case Design}

Traditional1y; a large effort is needed to develop test case buckets for a comprehensive test of all variations of program functions. The cause/effect graphs can be used to derive the design of such test case buckets. The programmer codes the graph and a tool produces the list of test cases needed for testing al1 causes and effects. Figure 14 shows what the tool would produce assuming the cause/effect graph shown in Figure 13:

The test bucket requires 6 test cases for having all causes invoked and all related effects observed.

\subsubsection{Integration \& Build Support}

The process of continuously integrating code and building drivers at different levels and possibly for a series of programing projects needs a complex tool to perform all steps in a controlled manner. Figure 15 shows the support functions and how they relate to each other. 
The programmer stores new code in a development library. He tests his code under a specific test system called driver. Upon test completion (successful) his code is integrated and stored in a master library. At that point, this code becomes available to other development groups.

In particular, test system or drivers are built from the master library and are then used by all development groups. Finally, the system to be released is build from the master library. Errors found during testing are fixed in the development library and, after successful testing the modified code is integrated into the master Iibrary. The whole integration and build process is fully controlled in that all actions are recorded and problems as for example unresolved dependencies are reported.

Preferrably, the described library system should be capable of supporting specification development as we11.

\section{$2.2 .5 \quad$ VM/370 (Figure 16)}

Testing needs a high amount of computing resources; testing system programs in particular requires different hardware configurations. VM/370 provides for the capability to have a variety of test systems running on the same installation. This makes the testing effort more flexible and economical.

\section{$2.3 \quad$ Management Aspects}

The previous sections dealt with the principals of the programming development process and the tools supporting it. I want to add some comments about managing this process. All techniques and tools, phase plans, and controls will not work unless programmers are involved in the implementation of the process. The programmers must do the detailed planning upon which the manager can base his overall plans. 
People are evaluated on the basis of the results they achieve whereby results are stated in terms of quality, timeliness, quantity, and cost. It should become common understanding, that recording and analyzing the above process measurements are part of a system programmers professional responsibility.

\subsubsection{Programming Teams}

Programmers of different skills are best organized in teams. Generally one to three teams report to a first-level manager. Each first-level manager has a librarian to support his teams. The roles of the team members and the librarien are shown in Figure 17 .

The team leader is responsible for preparing the functional specification and has technical responsibility for all design, logic specifications, and all code. He makes the technical decisions for the project. For most design, logic specifications, and code his job is to review and analyse the results produced by other programers. In this role, he supports the programmer, rather than evaluates him. (Evaluation of end results for specifications and code, and employee appraisals are done by the manager.) In addition, the team leader designs, writes logic specs, and codes the key elements of the product that his team is producing.

The co-team leader helps the team leader in all his duties and also codes key elements of the product. He will assume full team leadership responsibility if necessary. He is a technical peer of the team leader and his back up.

The programmers in the team are responsible for lower-level design and code, their own detailed planning, and testing. 
The librarian is an important part of the team. His job is to create, maintain, and own the library for the project. The project library includes both documentation and code. The 1ibrarian schedules and receives the runs from the computing center, and provides clerical services to the rest fo the group. The librarian is a full-fledged team member.

There are many advantages in programming teams. Senior people are directly and actively involved in the project. In the past these people would design the function and sometimes leave the project. Keeping senior people involved provides continuity. Since they generally produce work of higher quality, they can educate the junior members of the team. The team approach provides for more detailed and realistic plans.

\subsubsection{Forma1 Reviews}

During the last two years, there have been several projects which applied very formal review techniques to specifications, code, test cases, and publications. One review technique has been called Walkthru, another Inspection which word stems from a comon inspection practice in the world of hardware engineering. Both techniques are common in that a very detailed structured review, attended by the most knowledgeable and affected persons regarding system technology and dvelopment process, is performed. However, there is a difference between a Walkthru and an Inspection, in that only the latter emphasizes the recording and analysis of not only defects discovered but also development process measurments like number of defects by type.

The formal review is used by a developer as a resource to help him produce error-free products. The developer's attitude must be that other people are there to help, not to kill him with personal comments. The other participants (a moderator, the designer, 
other developer(s), a tester) must also realize that they are there to help. Managers have consciously to reinforce these feelings. Without this psychological atmosphere, formal reviews will not work. Formal reviews are not a management tool for evaluating programmers. The manager is not concerned about the number of problems found at this stage; just that they are found and corrected. The manager should use the number of problems as an indicator of existing or projected quality exposures.

Formal reviews are performed during phases II, III, and IV. Figure 18 shows the way a phase II walkthru works. The participants study the external specification and related material like cause/ effect graphs. They probe it for errors and develop a 1 ist of questions. In the meeting the material is thoroughly analyzed by all present. The issues are only recorded; the phase III walkthru concentrates on internal specifications and related material like HIPOs and Test Cases. The phase IV walkthru is a code walkthru. The length and the number of participants may vary. A review of an entire spec could involve eight people, a couple of days notice, and an offsite meeting. If relatively few changes have been made to an existing spec, the walkthru may involve only three people: the developer, his team leader, and the independent reviewer.

We have analyzed the effect of walkthrus on costs (Figure 19). The results showed that it was 14 to 15 times cheaper to find a problem during a walkthru than it was in unit test. And unit test is the point in the testing cycle at which it is cheap to find and fix an error.

3. CLOSING

We believe to have proof that the analysis and improvement of the programming development process has resulted in improved quality, i.e. fewer APARs relative to the released code (Figure 20). 
We are observing even better results with newly developed code. Figure 21 shows the APAR rate for the TSO scheduler code the rate of which is about one third the rate of code that consists in a conglomerate of old, changed and added code.

The same methods that increase quality have also a favorable effect on costs accounted over the whole process from inception until shipment of a system/component.

We hope that more and more system programming professionals will contribute new ideas on how to improve the total process of developing systems. This will be needed if new systems of growing size and increasing functional capability require another magnitude in manaing the development process which involves users, system programmers, and hardware engineers. 


\section{REFERENCES}

(1) W.B. Cammack and H.J. Rodgers, Ir. Improving the programming Process, IBM Technica1 Report 00.2483 (Oct. 73)

(2) W.R. Elmendorf

Cause-Effect Graphs in Functional Testing

IBM Technical Report 00.2487 (Nov. 73)

(3) F.T. Baker

System Quality through Structured Programming

1972 Fal1 Joint Computer Conference

(4) F.T. Baker

Chief Progranmer Team Management of Production Programing

IBM Systems Journal, Vol. II, Nov. 1, 1972

(5) H.D. Hitis

Top-Down Programing in Large Systems Courant Computer Sciences Symposium 1

New York Univ., June 1971

(6) P.W. Metzgex

Managing a Programming Project

Prentice-Hall 1973

(7) IBM HIPO Audio Education Package SR2O-9413 


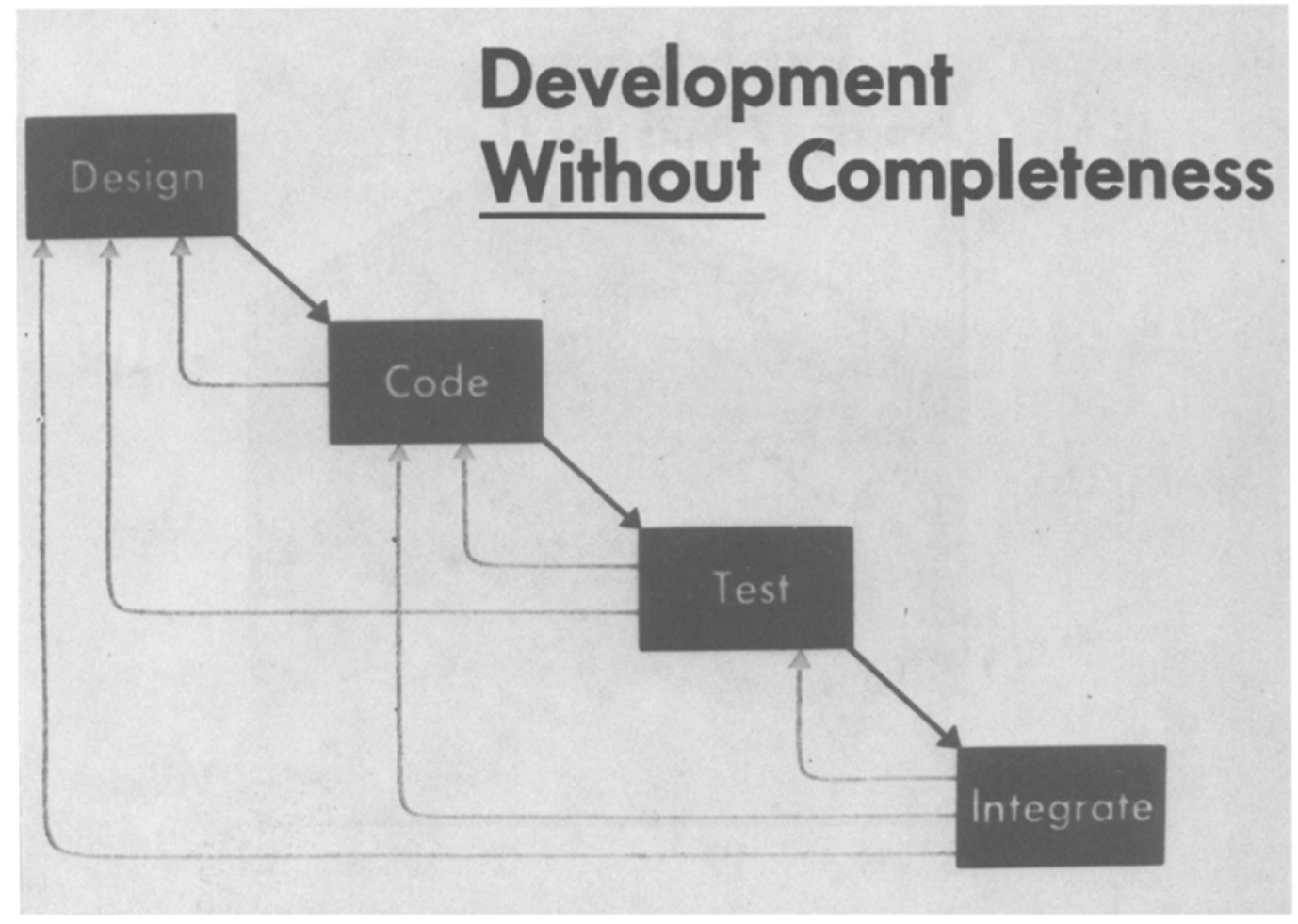

Fig. 1

\section{System build up}

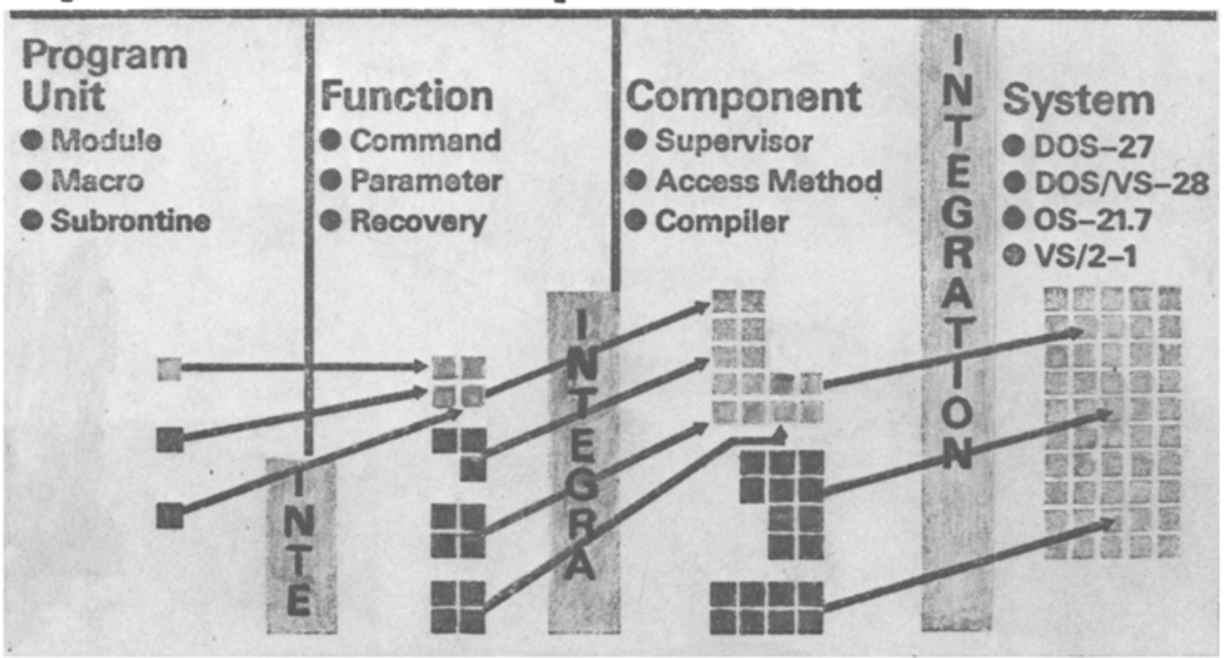

Fig. 2 


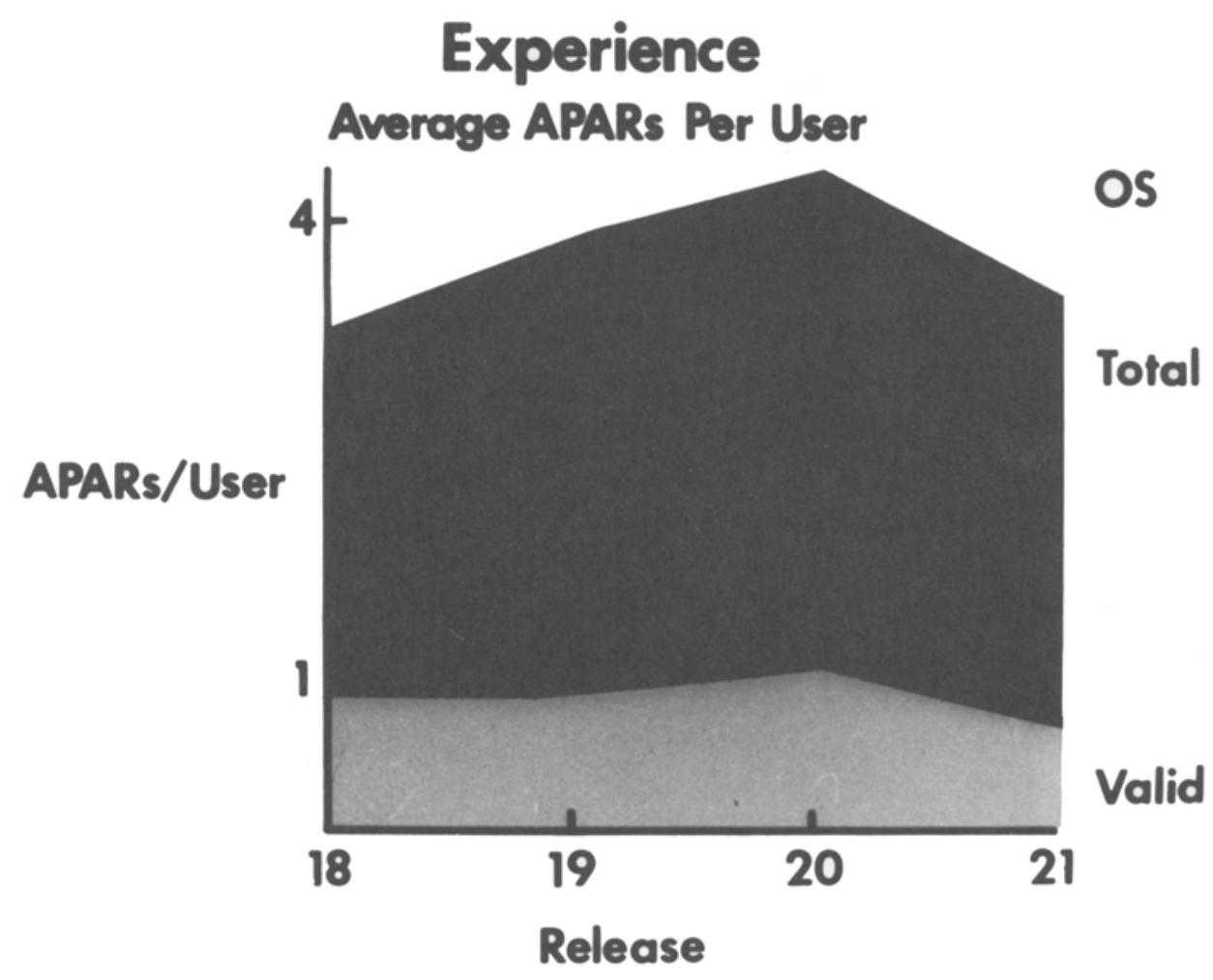

Fig. 3

Release 18 Costs

FCS +2 yrs. 


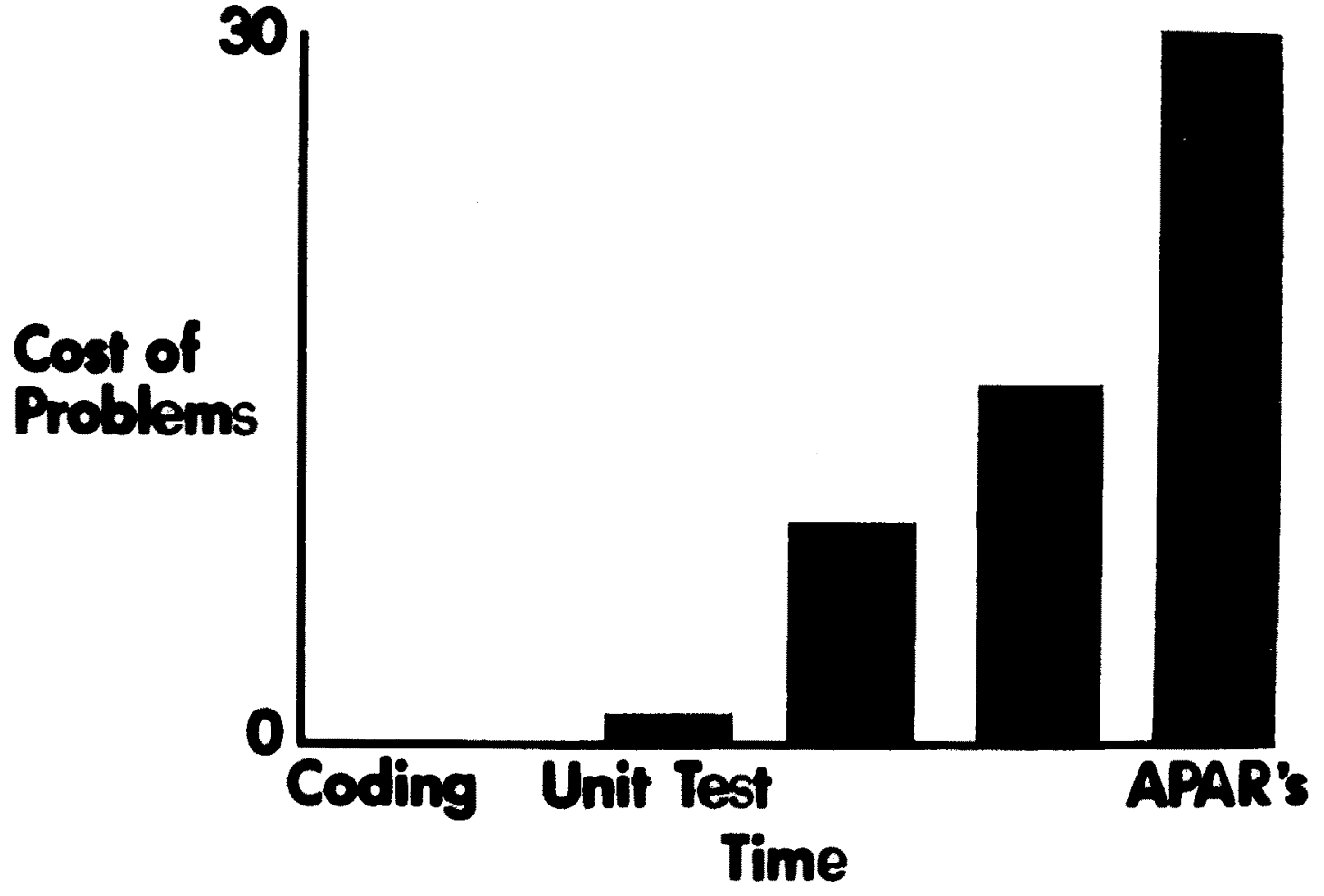

Fig. 5

Fig. 6 see page $30 \rightarrow$

Top-Down Design....

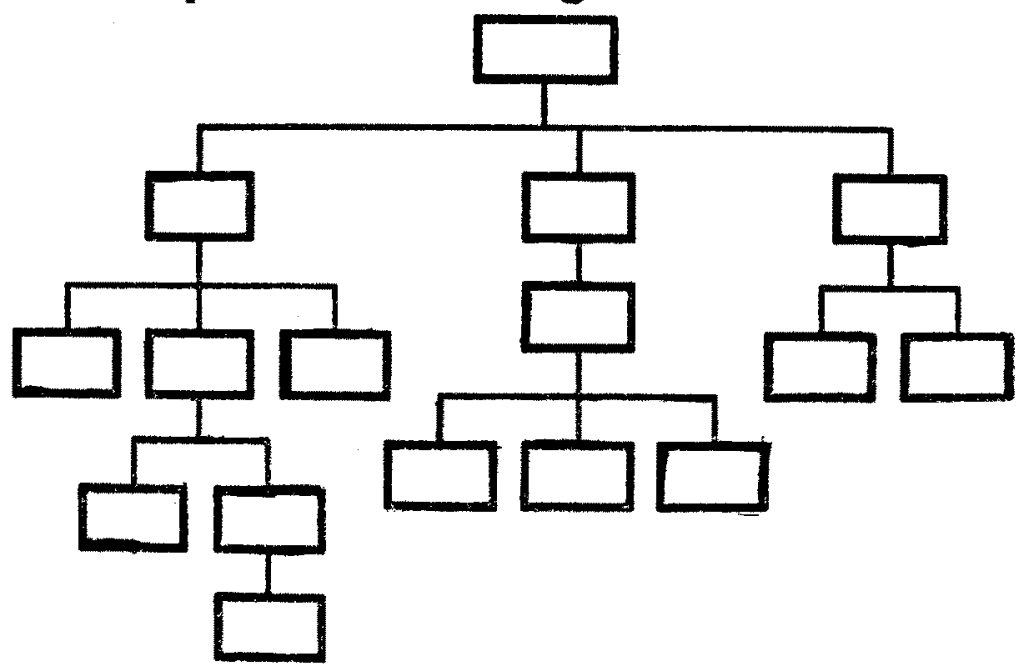

Fig. 7 


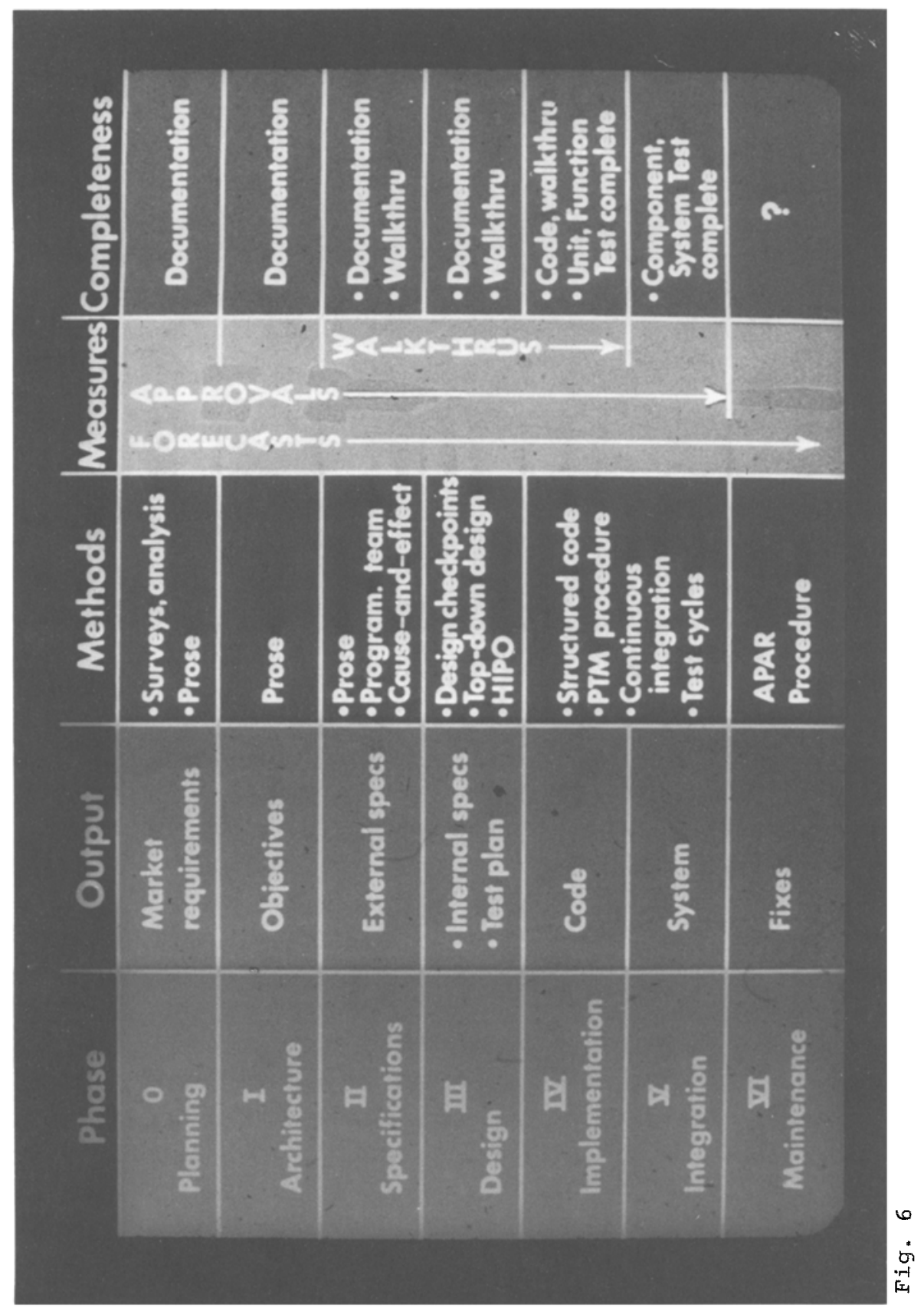




\section{Structured Programs}

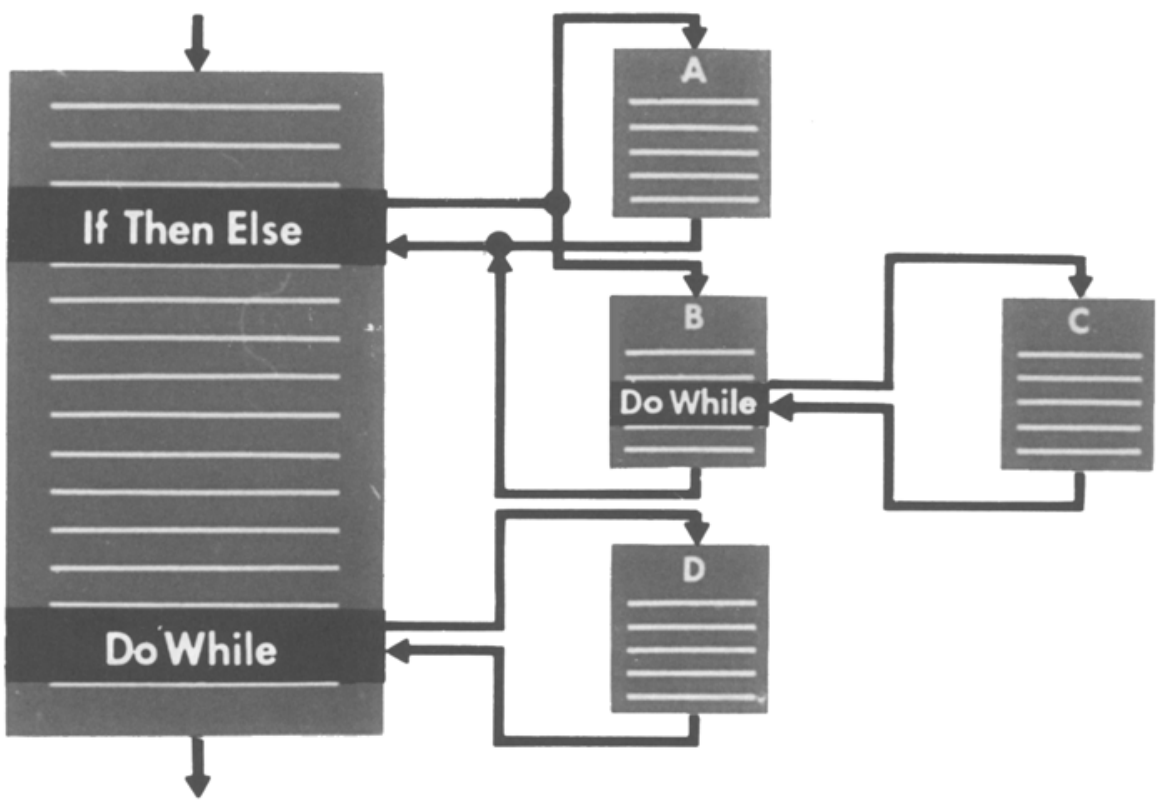

Fig. 8

\section{Structured Program Elements}
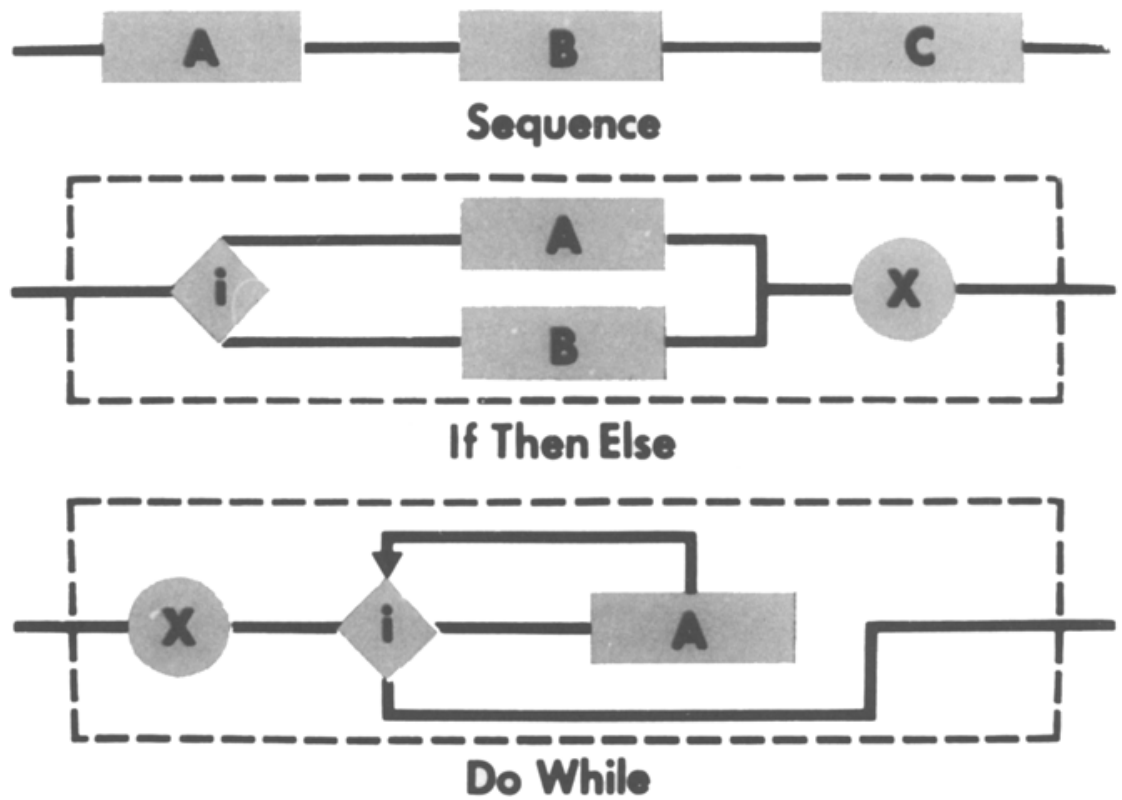

Fig. 9 


\section{Integration - As it Is}

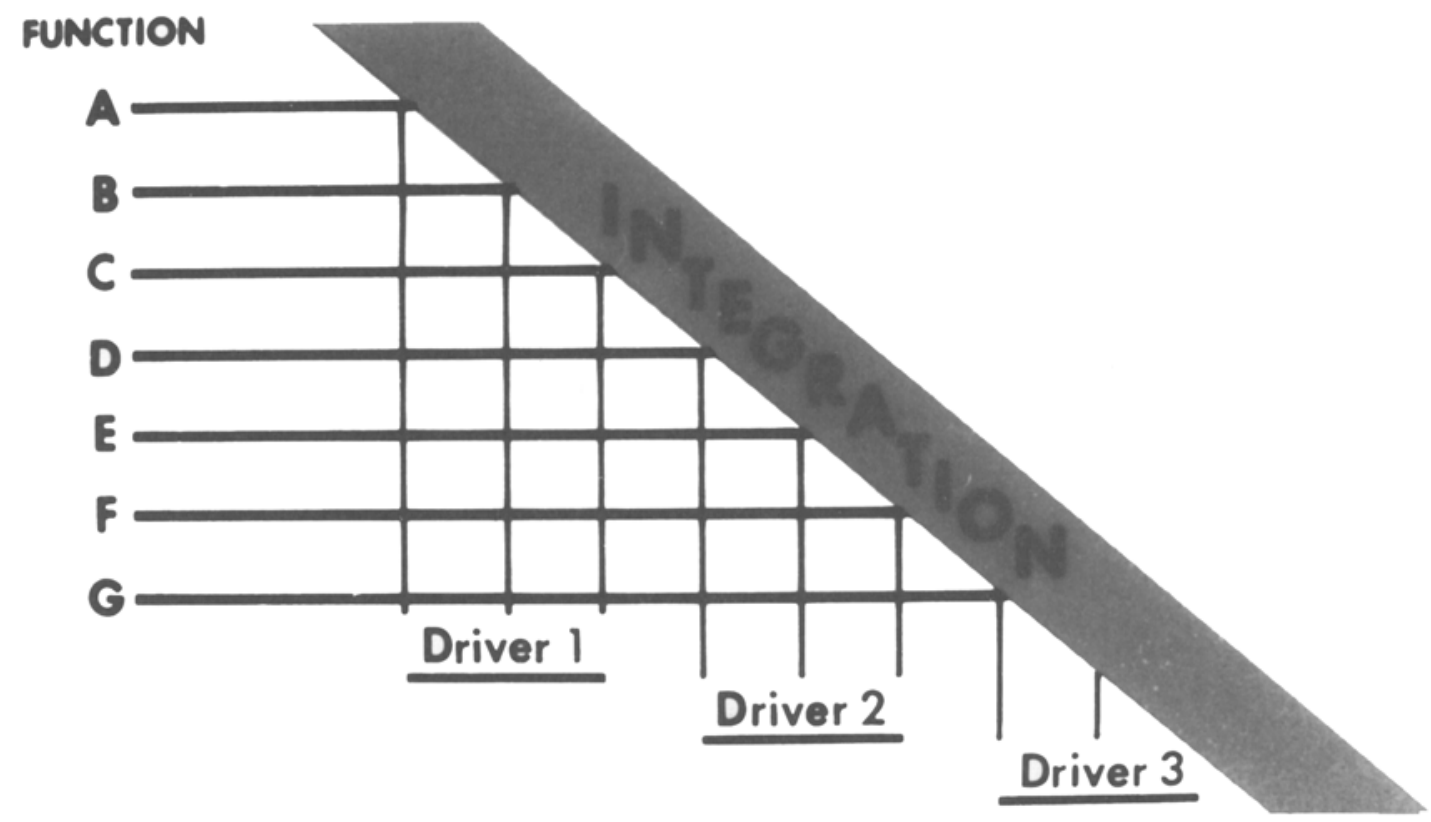

Fig. 10

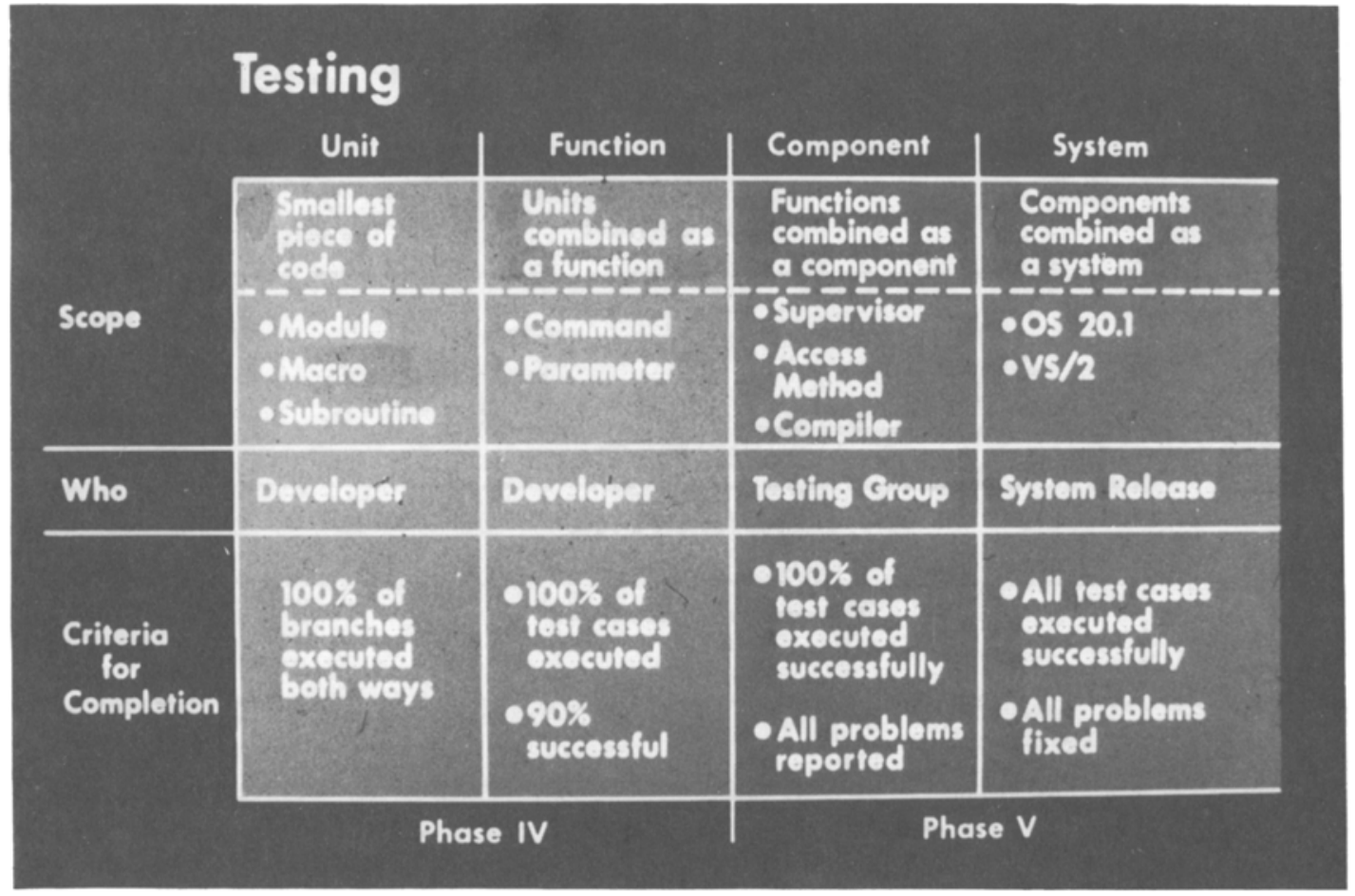




\section{HIPO}
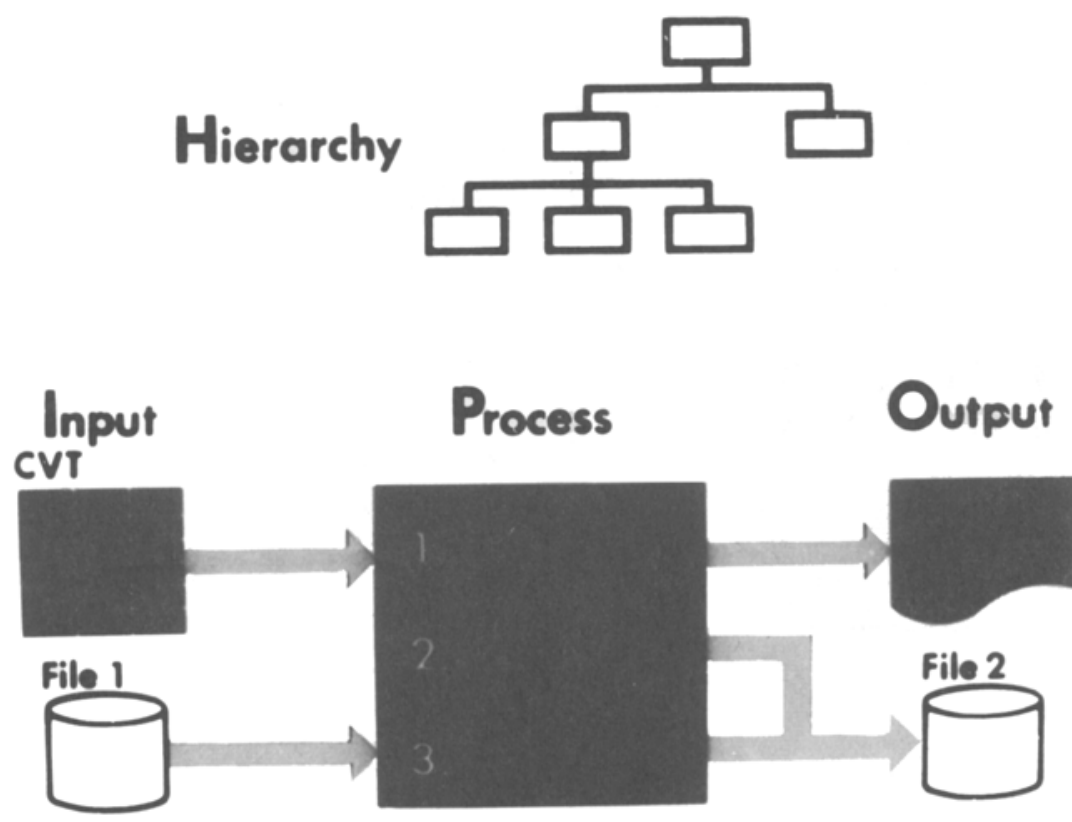

Draw the Graph

Nodes

1. 'OP1' is fixed binary

2. 'OP2 is fixed decimal

3. 'OPERATOR' is +

4. 'OPERATOR' is -

5. 'OP1' is imvalid

6. 'OP2' is imvalid

7. expreasion is valid

8. OPERATOR is valid

9. OPERATOR is invalid

Fig. 13

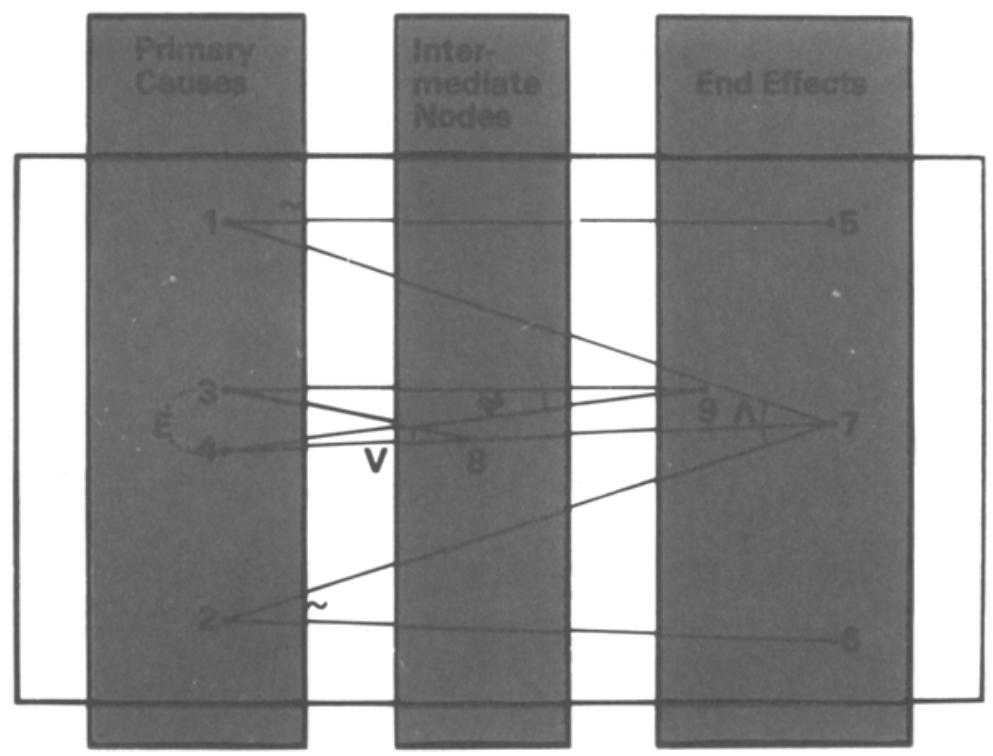




\section{The Test Case Library Design}

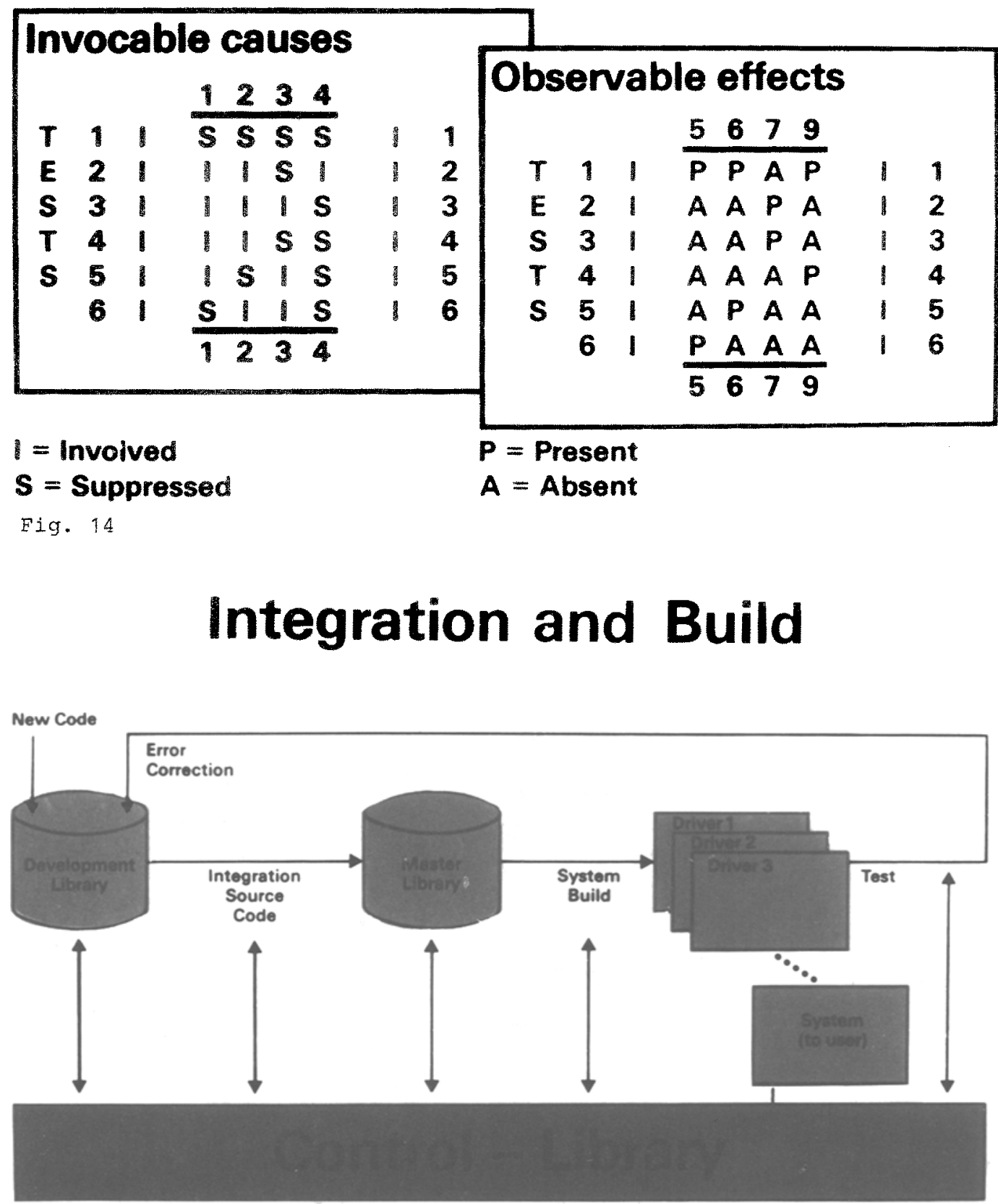

Fig. 15 


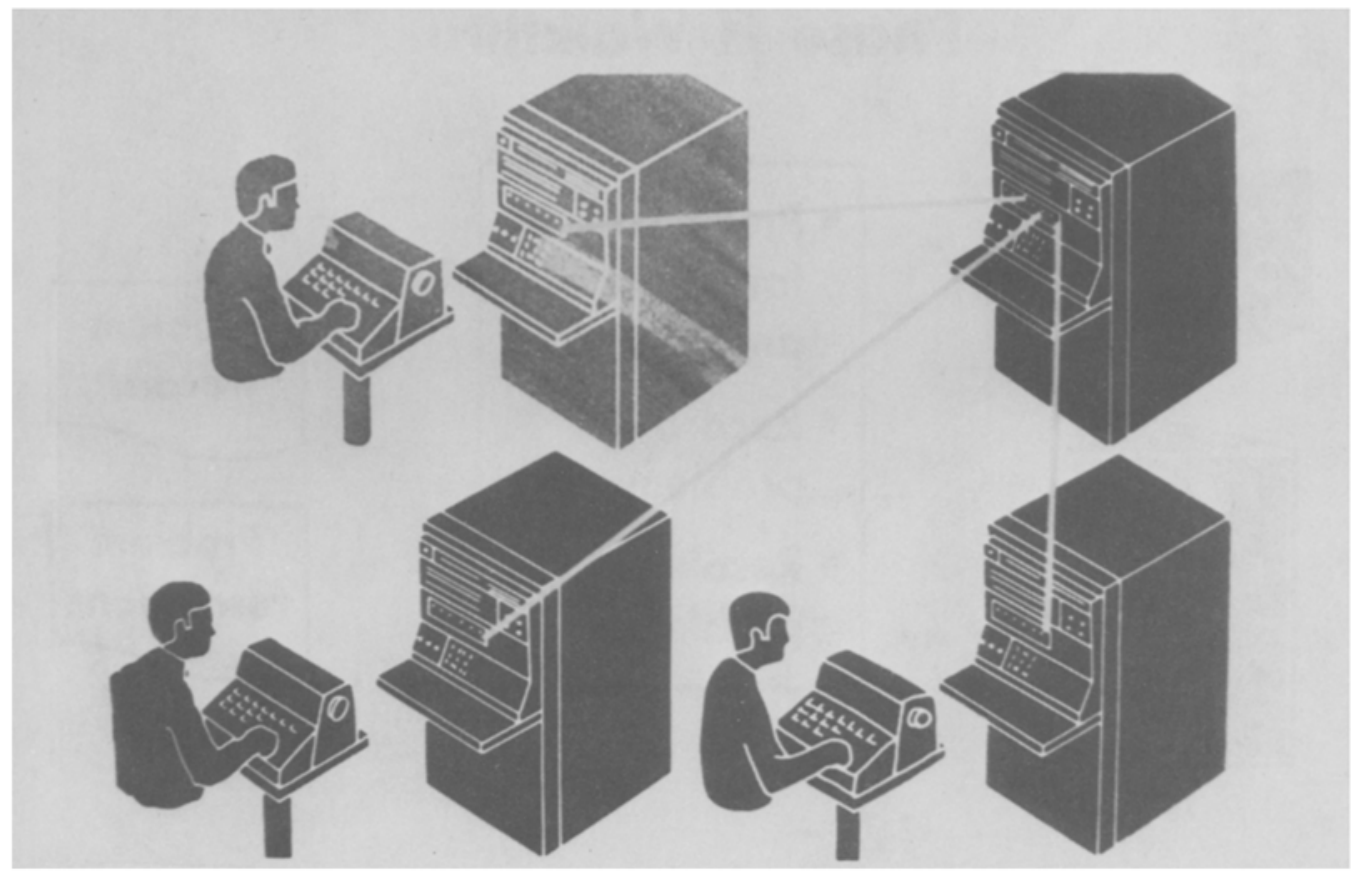

Fig. 16

\section{Programming Team}

\begin{tabular}{|c|c|}
\hline Team Leader & $\begin{array}{l}\text { - Makes major technical decisions } \\
\text { - Prepares functional specs } \\
\text { - Responsible for all design and code } \\
\text { - Codes key elements }\end{array}$ \\
\hline Co-Team Leader. & $\begin{array}{l}\text { Helps the feam leader } \\
\text { Codes key elements }\end{array}$ \\
\hline Brogrominers & $\begin{array}{l}\text { - Design and code } \\
\text { - Plan in detail } \\
\text { - Test at lower levels }\end{array}$ \\
\hline 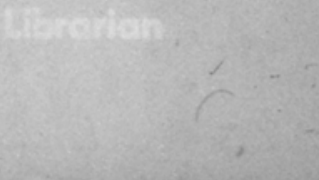 & $\begin{array}{l}\text { - Creates, maintains, and owns library } \\
\text { - Schedules and receives runs } \\
\text { - Provides clerical services }\end{array}$ \\
\hline
\end{tabular}

\section{Fig. 17?}




\section{Phase II Walkthru}

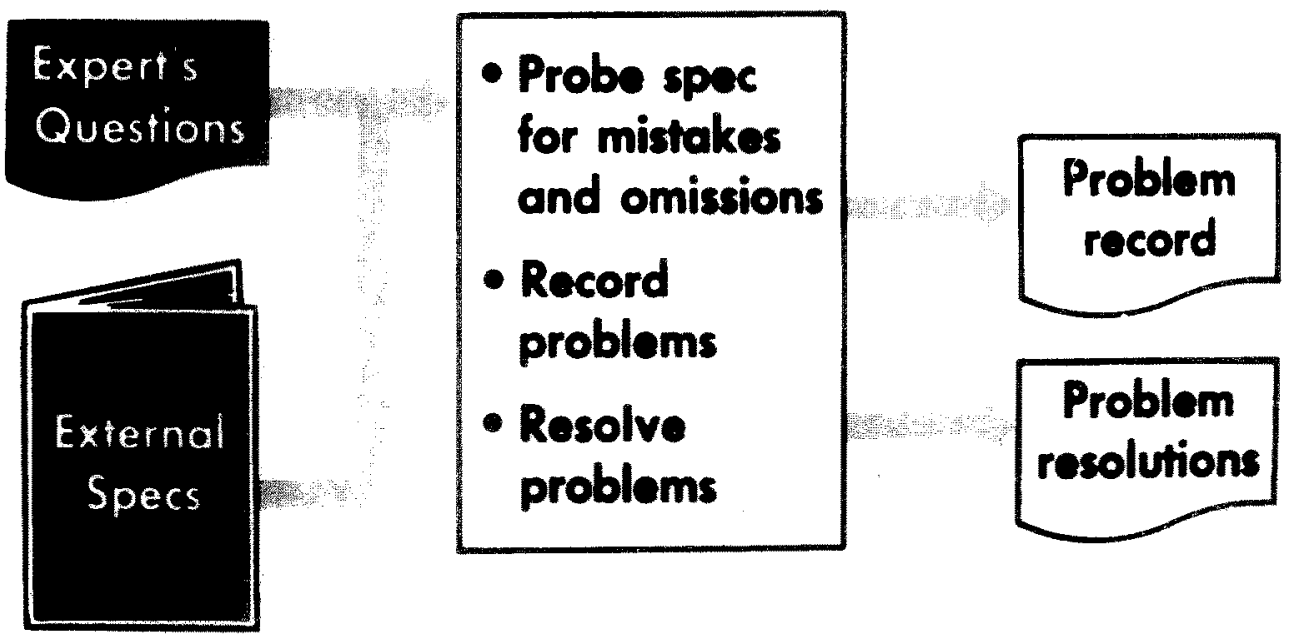

Fig. 18

Find Programming Errors Early

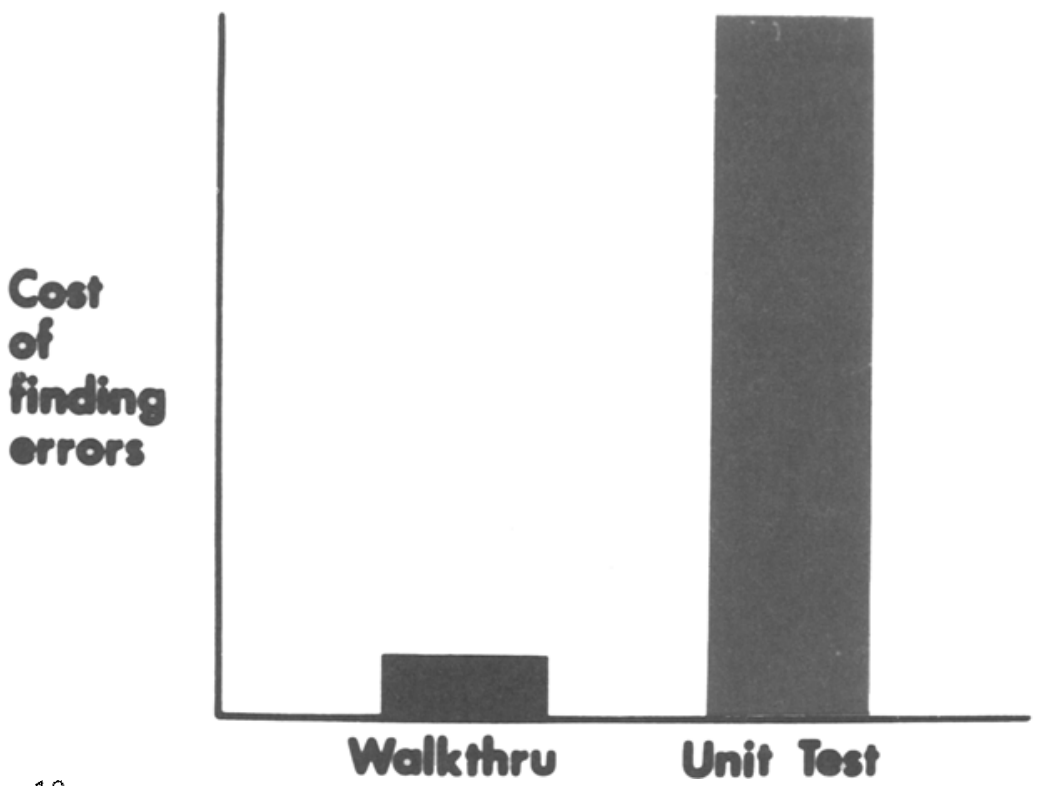

Fig. 19 
37

Results

Average ADARs Against Base Declining

Valid ADARs

Per Instruction

OS

18

19

20

21

Fig. 20

Release

Results

New Code is Higher Quality

ISO

Scheduler

Valid
A PARs

(normalized)

Fig. 21

Base Plus

New

Changed 